\title{
Aerobics Training for Athletes Limb Joints and Research on the Effects of the Characteristics of the Strength
}

\author{
Pengyu Zhao*
}

P.E. Department, JiLin Business and Technology College, Changchun 130507, Jilin, China

\begin{abstract}
Aerobic exercise is a "young" and loved by people of sports, therefore, in recent years research on aerobics is numerous, this article is on the basis of predecessors' research on aerobics athlete training body posture is analyzed and studied, for the study of the action process of knee joint force analysis provide the image of the space analytic foundation; The two 2-dof rigid body dynamics model was established, for the action process of knee joint torque analysis provides a theoretical basis; Later in this paper, by applying a table in the form of more clearly show the differences between the two aerobics athletes, through the comparison we can draw on the differences between two aerobics athlete was more obvious, therefore, for 1 calisthenics athletes need to shoulder joint control in 65 - degree is more appropriate, and sums up with the increase of the difficulty in the time-consuming, differences between two calisthenics athletes will be more and more obvious, at the same time reflects the calisthenics athletes 1 ability is prominent, through the comparison we found that the elbow of calisthenics athletes under than 12 aerobics athlete ability, therefore proves that the no. 1 aerobics athlete's comprehensive strength is strongest. Then by studying the aerobics athlete's habits are drawn in the Angle of minimum right knee joint position with a minimum right knee joint Angle position under the two positions when the joint Angle of the hour, the greater the moment will heavy torque, the greater the muscle torque will increase, the study will be the study of aerobics biomechanics analysis theory plays a certain role in promoting.
\end{abstract}

Keywords: Aerobics athlete, biomechanics, limb joints and muscle strength, physiological functions.

\section{INTRODUCTION}

Aerobics event belongs to non-Olympic Games events, aerobics development in China hasn't been accepted by people after going through a considerable long process, with people's living standards improvement, Chinese aerobics event are also entering into every civil mind, in the early period of the century, Chinese aerobics athletes participated in world aerobics championships, and achieved good result of ranking the seventh, and subsequent one year, in Chinese individual men competition, it got good result of ranking the top five, and in the process, Chinese aerobics have also gradually caught up with international pace, it let aerobics to gradually move towards maturity [1].

Regarding aerobics event research, many scholars and experts have carried out research, and meanwhile got abundant results, such as: Tan Yin-Yue in competitive aerobics event techniques analysis, took national aerobics men singles top eight athletes in 2005 as research objects to make research, the result showed that regarding jumping technical motion knee joint buffer range should be smaller than its supporting leg ankle joint buffer range, Chinese aerobics were to be improved in difficulty elements' air posture controlling, movement pattern control aspect, from which men were obviously weaker than women, so men aerobics athletes should more intensify in the aspect [2].
But women aerobics athlete during taking-off of difficulty element, they weren't in place as men aerobics athletes [3], Liu Hao had ever targeted at year 1994to 2004, three years frames aerobics evaluation rules, he made researches, finally got that aerobics techniques changes from beautification to competitiveness were mainly evolved by rules, and put forward higher level requirements on perfection of routine [4].

The paper just on the basis of above researches, targeted at aerobics athletes limb joints and muscle strength, it makes systematic researches, by applying biomechanics and to other methods, it makes analysis to provide theoretical guidance for aerobics athletes better performing their technology.

\section{BIOMECHANICAL ANALYSES OF AEROBICS ATHLETES LIMB JOINTS AND MUSCLE STRE- NGTH FEATURES}

Research based on aerobics can divide it as $a, b, c, d$ four kinds, from which the paper a kind totally includes Capoeira kind, Helicopter kind, Flair kind, cut kind, A-Frame kind, Plio push up kind, Wenson support kind, and push up kind. A-frame kind's series of motions totally contains seven kinds that are respectively explosive A-frame, explosive A-frame to Wenson, explosive A-frame twist to $1 / 2$, explosive Aframe twist to $1 / 2$ and then to Wenson, explosive A-frame twist to $1 / 2$ and then raise legs to Wenson, I arm explosive A-frame, I arm explosive A-frame and then to Wenson, in the whole process, knee part movement is relative frequent, in the following it makes research on knee force problems. 
Base on aerobics athletes biomechanics research, it can divide lower limbs force into low impact pace, high impact pace and non-impact pace three main kinds, from which low impact pace includes: marching type, touching step type, stepping forward type, one leg raising type; high impact pace: stepping forward and jumping type, two legs taking-off type, one leg taking-off type, back kicking and running type; non-impact pace. According to human body composition and attributes kinematics features, it can regard human body as rigid system model that is composed of 14 links, in the system, calculation the human body deducts right knee beneath joints weights can be regarded as total weight deducts right knee beneath two joints weights, and right knee beneath two joints weights total weight $G$ can be got by formula (1) calculating:

$G=m g \times\left(P_{i}+P_{j}\right)$

In formula (1), $m$ represents human body total mass, $g$ represents local gravity accelerated speed, $P_{i}, P_{j}$ are respectively right knee beneath two joints relative weights.

On the condition of human body balance, it can accord to mechanical conditions and force translation theorem, for aerobics training process knee force status, it makes analysis, as formula (2) shows relationships among muscle strength moment $N_{U}$, friction moment $N_{f}$, supporting moment $N_{n}$ and gravity moment $N_{m}$ that deducts right knee joint beneath joints.

$N_{U}+N_{f}+N_{n}=N_{m}$

The paper mainly researches human body knee joint force status in aerobics athletes training process, the parts that are correlated to knee joint is hip joint, thigh and shank, it can simplify human body lower limbs into plane two rigid bodies two freedom degree model, from which it gets involved in Hip, Thigh, Knee, Shank and ankle, apply Lagrange equation to establish constraint particle system dynamical equation, define Lagrange function $L$ as difference between system dynamic energy $K$ and potential energy $P$, as following:

$L=K-P$

In above formula $K, P$ can use any convenient coordinate system to express, system dynamic equation is as following formula shows :

$F_{i}=\frac{d}{d t}\left(\frac{\partial L}{\partial \dot{q}_{i}}-\frac{\partial L}{\partial q_{i}}\right) \quad i=1,2, \cdots, n$

In above formula, $q_{i}$ is dynamic energy and potential energy coordinate, $\dot{q}_{i}$ is corresponding speed, $F_{i}$ is the $i$ coordinate acted force, thigh and shank joint variables are respectively using intersection angles $\alpha_{1}, \alpha_{2}$ to express, hip joint and knee joint corresponding moments respectively use
$N_{h}, N_{k}$ to express, thigh and shank masses are respectively using $m_{1}, m_{2}$ to express, thigh and shank lengths are respectively $l_{1}, l_{2}$, thigh and shank mass center position and joint center's distances are respectively $p_{1}, p_{2}$, therefore it is clear that thigh mass center coordinate $\left(X_{1}, Y_{1}\right)$ is solving according to following formula, similarly shank mass center coordinate $\left(X_{2}, Y_{2}\right)$ can also be solved by applying same methods:

$\left\{\begin{array}{l}X_{1}=p_{1} \sin \alpha_{1} \quad Y_{1}=p_{1} \cos \varepsilon_{1} \\ X_{2}=l_{1} \sin \alpha_{1}+p_{2} \sin \left(\alpha_{1}+\alpha_{2}\right) \\ Y_{2}=-l_{1} \cos \alpha_{1}-p_{2} \cos \left(\alpha_{1}+\alpha_{2}\right)\end{array}\right.$

System dynamic energy $E_{k}$ and system potential energy $E_{p}$ expressions are as following formula shows:

$$
\left\{\begin{array}{l}
E_{k}=E_{k 1}+E_{k 2}, E_{k 1}=\frac{1}{2} m_{1} p_{1}^{2} \dot{\alpha}_{1}^{2} \\
E_{k 2}=\frac{1}{2} m_{2} l_{1}^{2} \dot{\alpha}_{1}^{2}+\frac{1}{2} m_{2} p_{2}^{2}\left(\dot{\alpha}_{1}+\dot{\alpha}_{2}\right)^{2}+m_{2} l_{2} p_{2}\left(\dot{\alpha}_{1}^{2}+\dot{\alpha}_{1} \dot{\alpha}_{2}\right) \cos \alpha_{2} \\
E_{p}=E_{p 1}+E_{p 2}, E_{p 1}=\frac{1}{2} m_{1} g p_{1}\left(1-\cos \alpha_{1}\right) \\
E_{p 2}=m_{2} g p_{2}\left[1-\cos \left(\alpha_{1}+\alpha_{2}\right)\right]+m_{2} g l_{1}\left(1-\cos \varepsilon_{1}\right)
\end{array}\right.
$$

By above formula, it is clear that above formula showed Lagrange function expression, by formula (3) showed system dynamic equation, it can get hip joint and knee joint torques $M_{h}$ and $M_{k}$ as following formula shows:

$$
\begin{aligned}
& {\left[\begin{array}{l}
M_{h} \\
M_{k}
\end{array}\right]=\left[\begin{array}{ll}
B_{11} & B_{12} \\
B_{21} & B_{22}
\end{array}\right]\left[\begin{array}{l}
\ddot{\alpha}_{1} \\
\ddot{\alpha}_{2}
\end{array}\right]+\left[\begin{array}{ll}
B_{111} & B_{122} \\
B_{211} & B_{222}
\end{array}\right]\left[\begin{array}{c}
\dot{\alpha}_{1}^{2} \\
\dot{\alpha}_{2}^{2}
\end{array}\right]+} \\
& {\left[\begin{array}{ll}
B_{112} & B_{121} \\
B_{212} & B_{221}
\end{array}\right]\left[\begin{array}{l}
\dot{\alpha}_{1} \dot{\alpha}_{2} \\
\dot{\alpha}_{2} \dot{\alpha}_{1}
\end{array}\right]+\left[\begin{array}{l}
B_{1} \\
B_{2}
\end{array}\right]}
\end{aligned}
$$

Among them, in above formula, $B_{i j k}$ expression is as following formula shows:

$$
\begin{aligned}
& B_{111}=0 \quad B_{222}=0 \quad B_{121}=0 \quad B_{22}=m_{2} p_{2}^{2} \\
& B_{11}=m_{1} p_{1}^{2}+m_{2} p_{2}^{2}+m_{2} l_{1}^{2}+2 m_{2} l_{1} p_{2} \cos \alpha_{2} \\
& B_{1}=\left(m_{1} p_{1}+m_{2} l_{1}\right) g \sin \alpha_{1}+m_{2} p_{2} g \sin \left(\alpha_{1}+\alpha_{2}\right) \\
& B_{12}=m_{2} p_{2}^{2}+m_{2} l_{1} p_{2} \cos \alpha_{2} \quad B_{21}=m_{2} p_{2}^{2}+m_{1} l_{1} p_{2} \cos \alpha_{2} \\
& B_{122}=-m_{2} l_{1} p_{2} \sin \alpha_{2} \quad B_{211}=m_{2} l_{1} p_{2} \sin \alpha_{2} \\
& \left.B_{112}=-2 m_{2} l_{1} p_{2} \sin \alpha_{2} \quad B_{212}=B_{122}+B_{211} \quad B_{2}=m_{2} p_{2} g \sin \left(\alpha_{1}+\alpha_{2}\right)\right]
\end{aligned}
$$

When aerobics athletes take training, hip joint is a key joint that decides A-frame kind and others series of movements' completion, hip joint movement is whole body gravity center that decides height and balance functions, besides to clearly express, we use a to represent Wenson posture, then use $\mathrm{b}$ to represent explosive A-frame twist to $1 / 2$ posture, use $\mathrm{c}$ to represent explosive A-frame to Wenson and then rotate to $1 / 2$, use $d$ to represent explosive A-frame posture, besides we also use an, bn, cn, dn to respectively express above four groups' left and right hip joints central 
Table 1. Two aerobics athletes hip joint parameters comparison (unit: $\mathbf{m m}$ ).

\begin{tabular}{|c|c|c|c|c|c|c|c|c|c|c|c|c|}
\hline Code No. & a Left & a Right & b Left & b Right & c Left & c Right & d Left & d Right & an & bn & cn & dn \\
\hline 1 & 1062.3 & 1052.1 & 944.6 & 1045.2 & 955.78 & 1014.2 & 956.7 & 977.4 & 1065.1 & 1029.8 & 1038.4 & 1015.9 \\
\hline 2 & 950.65 & 964.15 & 884.15 & 978.15 & 884.14 & 896.25 & 907.2 & 948.75 & 987.47 & 958.25 & 958.25 & 968.25 \\
\hline
\end{tabular}

Table 2. Two aerobics athletes' hip joint parameters movement comparison.

\begin{tabular}{|c|c|c|}
\hline & No. 1 Aerobics Athlete & No. 2 Aerobics Athlete \\
\cline { 2 - 3 } & $\bar{x} \pm S D$ & $884.152 \pm 26.145$ \\
\hline \hline a Left & $1061.124 \pm 10.998$ & $964.152 \pm 5.034$ \\
\hline a Right & $1099.452 \pm 4.258$ & $950.656 \pm 7.145$ \\
\hline b Left & $1052.014 \pm 1.845$ & $978.154 \pm 8.129$ \\
\hline b Right & $1092.592 \pm 18.987$ & $907.263 \pm 10.554$ \\
\hline c Left & $936.252 \pm 6.124$ & $896.256 \pm 16.425$ \\
\hline c Right & $974.147 \pm 2.048$ & $884.147 \pm 19.047$ \\
\hline d Left & $964.195 \pm 26.789$ & $948.753 \pm 10.841$ \\
\hline d Right & $958.544 \pm 6.481$ & $958.256 \pm 18.648$ \\
\hline an & $984.528 \pm 22.967$ & $958.256 \pm 17.109$ \\
\hline bn & $1088.413 \pm 18.642$ & $987.473 \pm 6.125$ \\
\hline cn & $1110.213 \pm 6.458$ & $968.257 \pm 5.314$ \\
\hline dn & $968.223 \pm 4.514$ & \\
\hline
\end{tabular}

point positions. When two aerobics athletes take training, hip joints $\mathrm{Z}$ axis positions' relative parameters are as following Table 1 show:

By above Table 1, we can get: in above two aerobics athletes training, in explosive A-frame twist $1 / 2$ to Wenson posture and explosive A-frame twist to $1 / 2$ these two movements, No. 1 aerobics athlete is best both in explosive Aframe to Wenson and explosive A-frame two movements' $3 D$ coordinates and $Z$ axis, and in two aerobics athletes training, regarding peak position hip joints comparative status is as Table $\mathbf{2}$ show:

By above Table 2, we know that when No. 1 and No. 2 aerobics athletes complete ab two groups of movements, there are no big differences between No. 1 and No. 2 aerobics athletes, which proves the two complete the two kind of movements have no difference; and cd two groups of movements exist obvious differences, in hip joint spring height aspect, No. 1 athlete is far higher than No. 2 athlete. When two aerobics athletes take training, peak hip joints comparison is as following Table $\mathbf{3}$ show:

By above Table 3, we can see that in comparison between No. 1 and No. 2 athletes, there are obviously differences from previous table, two people have very big differences in coordinate position, and in hip joint peak, No. 1 athlete is higher than No. 2 athlete but it is not especially obvious.
Regarding athlete should joint angles research, it mainly starts and ends with push up, it is about main exertion phase in push up phase, it can play balance roles, but it cannot last to movement completion, so the phase mainly analyzes joint angles' features and makes comparison, starting parameters are as following Table $\mathbf{4}$ show:

Ending phase shoulder joint correlation parameters analysis Table 5:

By above Table 5, we can get in starting moment, No. 1 and No. 2 two athletes' shoulder angle are less than $70^{\circ}$, and in ending moment No. 2 shoulder angles are slightly big and No. 2 left and right shoulder is not balance.

Elbow joint in general, it doesn't participate movement completion process as shoulder joint, but it participates in balance maintaining process, so carries out two athletes' comparison and analysis by following table, as following Table 6 show:

In order to more vividly highlight two aerobics athletes' differences problems, the paper makes use of bar chart form more clearly presenting mutual differences and connections, after that make analysis of angles parameters in ending moment, its result is as following Table 7 show:

By above Table 7, we can get that in ending moment, No. 2 aerobics athlete left elbow angle is quite small in $b$, $d$ two groups, and No. 1 aerobics athlete two directions' elbow joints angles are larger than that of No. 2 aerobics athlete, so 
Table 3. Two aerobics athletes hip joint comparison.

\begin{tabular}{|c|c|c|}
\hline Parameter & No. 1 Aerobics Athlete & No. 2 Aerobics Athlete \\
\hline a Right & $1099.452 \pm 4.257$ & $1052.126 \pm 20.450$ \\
\hline b Left & $1061.124 \pm 10.998$ & $944.64 \pm 10.624$ \\
\hline c Left & $964.195 \pm 26.789$ & $955.785 \pm 14.123$ \\
\hline c Right & $974.147 \pm 2.048$ & $1014.224 \pm 11.33$ \\
\hline d Left & $936.252 \pm 6.124$ & $956.787 \pm 2.451$ \\
\hline bn & $1088.413 \pm 18.642$ & $1029.852 \pm 11.575$ \\
\hline $\mathrm{cn}$ & $984.528 \pm 22.965$ & $1038.451 \pm 10.748$ \\
\hline $\mathrm{dn}$ & $968.223 \pm 4.514$ & $1015.974 \pm 7.546$ \\
\hline
\end{tabular}

Table 4. Aerobics athletes' starting moment should joint angles coefficients.

\begin{tabular}{|c|c|c|c|c|c|c|c|}
\hline Code No. & a Left & a Right & b Left & b Right & c Left & c Right & d Left \\
\hline \hline 1 & 82.12 & 78.05 & 79.40 & 76.27 & 78.56 & 76.11 \\
\hline 2 & 69.14 & 65.12 & 63.10 & 66.47 & 62.45 & 62.66 \\
\hline
\end{tabular}

Table 5. Aerobics athletes' ending moment should joint angles coefficients.

\begin{tabular}{|c|c|c|c|c|c|c|c|}
\hline Code No. & a Left & a Right & b Left & b Right & c Left & c Right & d Left Right \\
\hline \hline 1 & 76.00 & 82.10 & 82.60 & 56.82 & 76.12 & 75.84 & 86.96 \\
\hline 2 & 75.12 & 74.23 & 61.70 & 67.80 & 49.55 & 54.06 \\
\hline
\end{tabular}

Table 6. Aerobics athletes' starting moment elbow joint angles coefficients.

\begin{tabular}{|c|c|c|c|c|c|c|c|c|c|c|}
\hline Code No. & a Left & a Right & b Left & b Right & c Left & c Right Right & d Left & 68.20 & 61.92 \\
\hline \hline 1 & 68.40 & 66.25 & 70.21 & 63.25 & 62.05 & 75.84 & 66.89 \\
\hline 2 & 71.23 & 68.22 & 72.78 & 68.84 & 77.23 \\
\hline
\end{tabular}

Table 7. Aerobics athletes' ending moment elbow joint angles coefficients.

\begin{tabular}{|c|c|c|c|c|c|c|c|c|}
\hline Code No. & a Left & a Right & b Left & b Right & c Left & c Right & d Left & d Right \\
\hline \hline 1 & 68.85 & 64.27 & 68.01 & 60.35 & 63.42 & 64.78 & 63.29 & 56.87 \\
\hline 2 & 66.11 & 57.73 & 54.51 & 62.85 & 56.18 & 75.61 & 38.18 & 77.64 \\
\hline
\end{tabular}


Table 8. Aerobics athletes' habitual position posture quadriceps femoris weight loading status.

\begin{tabular}{|c|c|c|c|}
\hline Aerobics Athletes Code No. & $\begin{array}{c}\text { Quadriceps Femoris Muscle } \\
\text { Strength in Aerobics Athletes' } \\
\text { Habitual Position Posture }\end{array}$ & $\begin{array}{c}\text { Aerobics Athletes Themselves } \\
\text { Weight }\end{array}$ & Weight Bearing Ratios \\
\hline \hline 1 & 2093.72 & 558.60 & 3.75 \\
\hline 2 & 2227.50 & 697.76 & 3.19 \\
\hline 3 & 1840.92 & 534.10 & 3.14 \\
\hline 4 & 2002.43 & 457.13 & 3.36 \\
\hline 5 & 1521.87 & 567.91 & 4.09 \\
\hline 6 & 2322.18 & 553.21 & 2.25 \\
\hline 7 & 1243.78 & 641.90 & 3.37 \\
\hline 8 & 2160.51 & 509.11 & 3.05 \\
\hline 10 & 1552.32 & 406.21 & 3.40 \\
\hline Average value \pm standard deviation & $1834.68 \pm 383.76$ & $547.21 \pm 83.43$ & $3.35 \pm 0.48$ \\
\hline
\end{tabular}

No. 1 should contract more elbow joint angle regarding Aframe kind in starting moment.

During aerobics athletes training process, muscle is the main body that generates strength. When in standing position posture, human knee joint surrounding muscle maintains joint stability; it can guarantee human standing posture. It is well known that every individual standing posture has a certain difference so that make every individual muscle torque has also the difference. When aerobics athletes take training, they lie in the habitual posture, its right knee joint stability mainly relies on quadriceps femoris and ligament to maintain, and while knee joint makes flexion in habitual position, it will let quadriceps femoris excessive extension that prone to be fatigued, causing patellar ligament also prone to get injured.

In order to make research on knee joint muscle tolerable loading status when aerobics athletes take training, it get quadriceps femora's load bearing muscle strength values and their ratios with themselves own weight in habitual position posture as Table $\mathbf{8}$ shows.

By above Table $\mathbf{8}$ data, it is clear that knee joint in flexion, its quadriceps femoris muscle strength weight bearing ratios are in the range of $[2.25,4.09]$ times, arms force deceases by comparing with standing position posture, while muscle torque increasing leading quadriceps femoris muscle strength increases, meanwhile muscle strength increasing can cause quadriceps femoris weight bearing increases that let it prone to get tired.

Patellar ligament is the extension of quadriceps femoris tendon, quadriceps femoris extension would also accompany by patellar ligament extension, while quadriceps femoris extension will also accompany with patellar ligament extension, quadriceps muscle strength is basically also loaded by patellar ligament, so strengthen quadriceps femoris muscle strength and patellar ligament tractive tension is effective way to prevent injury.

\section{CONCLUSION}

The paper firstly introduces body postures status when aerobics athletes take training to provide space analysis image base for researching its motion process knee joint force analysis; establishes two rigid bodies two freedom degrees dynamical model to provide theoretical basis for motion process knee joint moment analysis; after that, the paper through applying table form, it more clear presents two aerobics athletes existing differences, by comparing, we can get that two aerobics athletes differences are relative obvious, so for No. 1 aerobics athlete, he should control shoulder joint in that is suitable, and in consumed time, it summarizes with difficulties increasing, two aerobics athletes differences will be more obvious, and meanwhile it reflects No. 1 aerobics athlete ability is relative prominent, by elbow joint aspect comparing, we find that No. 1 aerobics athlete ability is stronger than No. 2 aerobics athlete, so it proves No. 1 aerobics athlete comprehensive strength is stronger. Finally by researching aerobics athletes body right knee minimum joint angle, it gets habitual posture and right knee minimum joint angle instant posture such two postures, when joint angle gets smaller, muscle strength moment will be bigger, and gravity moment will be bigger, and muscle strength moment will accordingly increase.

\section{CONFLICT OF INTEREST}

The authors confirm that this article content has no conflict of interest.

\section{ACKNOWLEDGEMENTS}

Declared none. 


\section{REFERENCES}

[1] W. Kun, and Z. Min, "Analysis of competitive aerobics group horizontal supporting type difficulty element techniques and muscle activity features", Journal of Xian Physical Education University, vol. 27, no. 06, pp. 716-728, 2010.

[2] Z. Ming-Jun, and W. Qing-Sheng, "Flatfoot sports shoes designing sports biomechanical features analysis", Journal of Huizhou College, vol. 31, no. 06, pp. 102-105, 2011.
[3] Y. Hong, "Competitive aerobics straddle jump to push up threedimensional kinematics analysis", Journal of Military Sports College of Further Education, vol. 30, no. 01, pp. 65-69, 2011.

[4] L. Xing-Guan, "Application of sports biomechanics in aerobics", Journal of Nanjing Sport Institute (Natural Sciences), vol. 07, no. 04, pp. 36-39, 2008.

Received: June 10, 2015

Revised: July 29,2015

Accepted: August 15,2015

(C) Pengyu Zhao; Licensee Bentham Open.

This is an open access article licensed under the terms of the (https://creativecommons.org/licenses/by/4.0/legalcode), which permits unrestricted, noncommercial use, distribution and reproduction in any medium, provided the work is properly cited. 